\title{
Percepción de los docentes chilenos acerca del programa de escuelas focalizadas: elementos para el análisis de una política educativa de 'acción positiva'
}

Miguel Bustamante U.

Sebastián Donoso D.

Universidad de Talca

\begin{abstract}
Resumo
El Programa de Escuelas Focalizadas (antes P-900) es una iniciativa educativa de larga data impulsada en Chile por el Ministerio de Educación tras el retorno de la democracia (1990). Es uno de los programas que tuvo mayor éxito, aunque su rediseño se hace inminente por los resultados derivados de diversos estudios. Su estrategia significó apoyar pedagógicamente a las escuelas cuyos resultados en las pruebas de medición de logros de los cuartos grados se situaban en el 10\% con peores valores (actualmente en el 18\%). El proceso pedagógico sustentado por el Programa se centra en maestros y alumnos, con una acción positiva en diversos ámbitos. Los resultados dan cuenta de la percepción de los docentes participantes en una de las áreas geográficas con más bajos resultados y mayores índices de pobreza del Chile: la Provincia de Talca. Se trabajó con 249 docentes, aplicándoles un cuestionario estructurado con escalas likert y Nominal. Los resultados confirman que los docentes conocen el programa, que tienen una percepción positiva pero que sus nuevas prácticas aún no son valoradas como un factor de desarrollo laboral de significación. Desde el punto de vista de una política educativa de acción positiva es indispensable introducir cambios de significación que implican su rediseño, aspecto que se debate en las conclusiones del texto.
\end{abstract}

\section{Palavras-chave}

Innovación docente - Escuelas focalizadas - Percepción docente Política educacional de acción positiva.
Correspondência:

Sebastián Donoso D.

Universidad de Talca

Casilla 747 - Talca - CHILE

e-mail: sdonoso@utalca.cl 


\section{The perception of Chilean teachers about the program of focused schools: elements for the analysis of a 'positive action' education policy}

Miguel Bustamante U. Sebastián Donoso D.

Universidad de Talca

\begin{abstract}
The Program of Focused Schools (formerly P-900) is a longstanding education initiative promoted in Chile by the Ministry for Education in the wake of the return to democracy (1990). It is one of the most successful programs, even if its redesign is already needed in face of results derived from several studies. Its strategy was to give pedagogical support to the schools whose pupils from the first four grades were within the 10\% worst results in assessment exams (nowadays the 18\% worst results). The pedagogical process proposed by the Program centers on teachers and pupils, with a positive action in several spheres. The text reports on the perception of participating teachers from one of the geographical areas with the worst results and levels of poverty in Chile: the Talca Province. The work involved 249 teachers, with the application of structured questionnaires with Likert and Nominal scales. The results confirm that the teachers know about the program, that they have a positive perception of $i t$, but that its new practices are still not valued as a significant factor of professional development. From the point of view of an education policy based on positive action it is essential to introduce changes of meaning, implying in the redesign of the program, an aspect discussed in the conclusions of the article.
\end{abstract}

\section{Keywords}

Innovation in teaching - Focused schools - Teacher perception Positive action education policy.
Contact:

Sebastián Donoso D. Universidad de Talca

Casilla 747 - Talca - CHILE

e-mail: sdonoso@utalca.cl 


\section{Antecedentes ${ }^{1}$}

El advenimiento de la democracia en Chile, desde inicios del año 90, tras casi 18 años de Gobierno Militar, dio origen a cambios significativos en el ámbito educacional. Uno de los primeros en implementarse y que ha seguido operando hasta la fecha, es el Programa de Escuelas Focalizadas, identificado en sus inicios como P-900, sigla con la que permaneció operando por más de una década. El P-900 apuesta a reforzar la docencia y a los docentes, con materiales adecuados para maestros y alumnos, apoyo familiar y una serie de beneficios dirigidos a mitigar el impacto de las diferencias socioeconómicas sobre los aprendizajes de los estudiantes.

La estrategia de focalización de las acciones en educación tiene una larga tradición en este campo, inicialmente fue asumida para tratar casos que estaban "fuera de la norma", a saber: grupos especiales con discapacidades físicas, mentales y conductuales, a los que se sumaron después otras visiones de grupos específicos bajo la concepción de lo que hoy se conoce como acción positiva. En esencia, comparten la comprensión y luego aceptación que ante determinadas circunstancias y condiciones deben asumirse procedimientos de trabajo educativo propios o particulares, dado que los modos de trabajo convencional no resultan eficientes sea por la magnitud del problema, los recursos involucrados para acometer la tarea, como finalmente por la velocidad de nivelación de esta población que requiere de mayor tiempo para reducir las diferencias con la población "normal” o común.

El P-900² fue ideado por los equipos técnicos que asumirían la conducción del Gobierno, hacia fines del año 1989. Entonces el diagnóstico de la situación se basó en cuatro ideas fuerza. Primero, que habían fuertes expectativas sociales sobre la educación por su rol como agente de movilidad social. Segundo, que el acceso al sistema se encuentra garantizado para la gran mayoría de niños y jóvenes. Tercero, que los incrementos de escolaridad no implican directamente au- mento de ingresos ni de empleabilidad de la población, y cuarto, que el paso de la escuela al liceo no asegura la adquisición de saberes útiles para la vida y participación en la sociedad (Sotomayor, 1999).

Estas ideas se asociaban a los resultados del sistema escolar que hacia 1988 mostraban en las pruebas de Matemática y Lenguaje de $4^{\circ}$ año básico, estándares de logro promedio levemente superiores al 50\%. Adicionalmente, se manifestaban dificultades importantes en retención de estudiantes, lo que se traducía en importantes tasas de deserción y repitencia. Estos resultados estaban fuerte y negativamente asociados con los sectores sociales más desfavorecidos, dando cuenta de la necesidad de generar un programa focalizado, de atención al segmento más necesitado de la sociedad, de acuerdo con la concepción de equidad asumida como discriminación positiva (Sotomayor, 1999).

La idea de llevar a cabo un programa en estos términos se basó en una experiencia de Talleres de Aprendizajes, desarrollados por algunas ONGs chilenas durante la segunda mitad de la década de los 70 y parte de los 80 , con niños jóvenes de sectores sociales pobres, como una forma de apoyo para su reinserción escolar.

\section{El Programa de Escuelas Focalizadas Completas}

El P-900 fue el Programa más relevante impulsado en el primer año del Gobierno democrático (1990-1991). Ello debido a que no se dispuso de recursos adicionales para impulsar nuevas iniciativas dado que el Gobierno militar había dejado un presupuesto para el sector educación el año 1990 equivalente al 75\% del correspondiente a 1980 (González, 2003), esto

1. Agradecemos a los memoristas Ingenieros Comerciales Srta. Marisol Andrea Rubio González y Sr. Pablo Hernán Acuña Rojas, el trabajo de campo realizado.

2. Este nombre surgió porque la población de escuelas correspondía al $10 \%$ inferior de los resultados de las pruebas de logro de Matemática y Lenguaje de $4^{\circ}$ año básico del país, cuyo número absoluto se aproximó a 900 establecimientos, aunque desde el comienzo fue siempre superior. 
implicó que al menos en ese primer año, el P-900 fuera implementado con el aporte financiero de la comunidad internacional. Desde entonces esta iniciativa se constituyó en un programa relevante y sus resultados han sido evaluados satisfactoriamente por el Estado (Dipres, 2001), como también por instituciones internacionales (OCDE, 2004).

El propósito central del P-900 es "Mejorar la calidad de los aprendizajes cognitivos y socioafectivos, de los alumnos/as de $2^{\circ}$ nivel de transición (preescolar) a $8^{\circ}$ básico, de las escuelas subvencionadas ubicadas en la franja del 10\% inferior de los resultados de las Pruebas que miden Calidad (logros) de la educación" (identificadas como pruebas $\mathrm{SIMCE}^{3}$ ). El segmento del 10\% inferior corresponde a los establecimientos que se incorporan al P-900, cifra utilizada hasta el año 2000. A partir del año 2001 se amplió la cobertura al 18\% inferior (DIPRES, 2001, p. 1).

El Programa entrega apoyo técnico y material especial a los establecimientos escolares participantes. Si bien tras diversas evaluaciones el diseño estratégico del Programa ha experimentado cambios, en lo fundamental para mejorar sus resultados, en esencia sigue los mismos principios

[...] fortalecer las destrezas culturales de base en los alumnos de estas escuelas, para lo cual se implementa una estrategia especial de apoyo material, de perfeccionamiento docente y de reforzamiento pedagógico a los niños con mayor retraso escolar. (Sotomayor, 1999, p. 71)

El programa se organiza en cinco componentes: Primero, desarrollo profesional docente para mejorar las prácticas pedagógicas mediante talleres, reuniones técnicas y la conformación del equipo de gestión educativa. Segundo, desarrollo de talleres de aprendizaje con monitores de la comunidad, a niños de $1^{\circ}$ a $4^{\circ}$ grado que tienen retraso escolar. Tercer componente, implementar la gestión educativa, que busca fortalecer esta dimensión en sus aspectos internos y externos, con sostenedores y autoridades. Cuarto compo- nente, profundizar la relación familia/escuela, que persigue fortalecer el conocimiento y apoyo mutuo, mediante diversas actividades dirigidas a mejorar los resultados de los niños. Finalmente, el quinto componente es proveer de material educativo: guias, textos complementarios, material didáctico etc. para alumnos, profesores y monitores (Dipres, 2001).

Pese a las distintas estrategias implementadas en el Programa, sus principales pilares son

[...] los Talleres de Perfeccionamiento Docente en servicio, la distribución de materiales educativos y bibliotecas de aula, y el apoyo a alumnos con rezago a través del apoyo de monitores de la comunidad. (Aylwin, 2003, p. 154)

La Reforma Educacional Chilena asumió al P-900 como uno de sus ejes de trabajo, proceso que permanece vigente aunque a partir del año 2001 lleva el nombre de Programa de Escuelas Focalizadas y tiene una cobertura del 18\% de los establecimientos con más bajos resultados en las pruebas SIMCE ${ }^{4}$.

Los resultados de aprendizaje de las escuelas básicas que forman parte de los dos programas focalizados, P900 y Educación Rural mejoraron consistente y más que en el resto del sistema escolar durante la década, reduciendo así la brecha entre los resultados de aprendizaje (correspondiente al 15\% más pobre de la matrícula) y al promedio del país. [...] los resultados de aprendizaje de los $4^{\circ}$ básicos de las escuelas del P900 en lenguaje, matemática y comprensión del medio natural, social y cultural, aumentaron más entre 1992 y 2002 que en el resto de las escuelas. (OCDE, 2003, p. 44)

3. Sistema de Medición de la Calidad de la Educación, SIMCE, estructura de instrumentos, entre ellas pruebas aplicadas a estudiantes del $4^{\circ}, 8^{\circ} \mathrm{y}$ $10^{\circ}$ grado del sistema educativo chileno.

4. Algunos textos definen el P-900 como el programa que trabaja con las escuelas más pobres, la confusión se debe al isomorfismo que se da entre las escuelas que tienen bajos resultados escolares con aquellas que atienden la población más pobre, de allí la importancia de comprender que se refiere a la situación de logros de aprendizaje de sus estudiantes. 
Pese a que los resultados del P-900 son positivos e importantes, se muestran aún insuficientes y están por debajo de los promedios nacionales.

Ello nos lleva a pensar que para estos establecimientos sigue siendo necesario entregar un marco de orientaciones claro y apoyo bastante acotado desde el centro, lo que deberá traducirse en buenos textos o materiales educativos, en una supervisión sistemática y de alta calidad técnica. Hacer esto con un foco puesto en el fortalecimiento de las escuelas es fundamental, de lo contrario este apoyo se eternizará. (GarcíaHuidobro; Sotomayor, 2003, p. 311)

Este comentario tiene sentido ya que los resultados del SIMCE sólo son comparables a partir del año 1996 en adelante. Lo que debe llamar a la cautela cuando se trata de generalizar sus resultados.

En el plano organizacional si bien muestra un buen grado de articulación con otros programas del Ministerio, la opinión de la DIPRES establece que las pautas de evaluación de los supervisores son muy generales, que no se logra el nivel de involucramiento adecuado de los sostenedores, ni tampoco hay una estrategia de coordinación sistemática con los Departamentos de Educación de cada municipio, y que la información recabada de las escuelas no ha sido debidamente usada por el Programa. Por otra parte, la evaluación reconoce que es un programa eficiente, habiendo mejorado los resultados escolares, sin embargo a nivel de egreso de los estudiantes las escuelas muestran algunas dificultades

[...] un 43\% de las escuelas que han egresado del programa presentaron una disminución relativa en el puntaje SIMCE respecto de escuelas de características similares en los años inmediatamente después de su salida. Por otra parte, el programa registra un número importante de escuelas que reingresan (39\% en el período 1994-1999) sin conocer con precisión sus causas. (Dipres, 2001, p. 5-6)
Como se establece, una de las claves del programa es el cambio de las prácticas docentes en aula. "La hipótesis central en esta etapa apunta al efecto del aula en la mala calidad de los aprendizajes de los alumnos" (Sotomayor, 1999, p. 75), a ella se responde con los "Talleres de Perfeccionamiento Docente" que han sido una herramienta clave en el programa. Esta estrategia se tuvo que adecuar el año 1998, al extenderse el programa a los cursos de $5^{\circ}$ a $8^{\circ}$ grado. Ello implicó desarrollar los talleres como reuniones técnicas de profesores, adquiriendo ese nombre y también el sentido de intercambio de experiencias.

\section{Los establecimientos escolares de la Provincia de Talca}

Al año 2004, la Provincia de Talca es predominantemente urbana aunque posee una importante participación de población rural que duplica y algo más la tasa promedio país de este sector. Pese a esta condición de mayor presencia de población escolar rural, que se asocia a cierto desmedro educativo, sus indicadores en este campo muestran en muchos aspectos semejanza con los estándares nacionales promedio, como en la misma cobertura del sistema de educación total que es un 5\% menor que la tasa promedio país en cobertura de la educación media (www.sinim.cl).

De igual forma, muestra cierta semejanza entre la cobertura de matrícula que tiene el sector municipal, la que se eleva un par de puntos sobre el 50\% de la matrícula total. Los indicadores de eficiencia interna del sistema, como supervivencia escolar, promoción, abandono y repitencia son equivalentes al promedio país con algunas diferencias menores de carácter no significativo. El problema del sistema educativo chileno no se encuentra en estas variables que se refieren al rendimiento intra-sistema, sino en los indicadores de calidad del proceso educativo.

La provincia de Talca cuenta con 50 Escuelas Focalizadas Completas, lo que representa algo menos del 25\% del total de establecimientos del territorio. En los últimos años se ha producido 
tanto en la provincia de Talca como en la Región del Maule (área geográfica superior que incluye a esta y otras tres provincias más), una notoria diferencia en los resultados educacionales medidos por el SIMCE entre las escuelas públicas y privadas, fenómeno ratificado en la medición del año 2005. Por este motivo, el gobierno implementó el programa Escuelas Focalizadas Completas, con la finalidad de acortar esta brecha. Éste se enfoca principalmente en las acciones de los docentes, debido a que son el agente clave para llevar a cabo las mejoras en la educación.

\section{Objetivo del estudio y el enfoque de la percepción}

El propósito del estudio fue determinar la percepción de los docentes participantes en el P-900 de la provincia de Talca, respecto del programa, para lo cual fue necesario establecer el grado de conocimiento del mismo y de sus metodologías empleadas identificar sus atributos y falencias, y precisar su grado de eficacia, identificando cómo influyen algunas variables en la percepción de los docentes.

Atendiendo que la percepción es el campo temático a partir del cual el estudio ordenará la información, el análisis desarrollado considera la visión de Robbins, sobre la percepción "como un proceso mediante el cual, los individuos organizan e interpretan sus impresiones sensoriales con el fin de darle significado a su ambiente" (1998, p. 90), visión complementada por Lambin:

[...] proceso por el cual un individuo selecciona e interpreta la información a la cual está expuesto. Los individuos tendrán, pues, en general, percepciones distintas de una misma situación, porque la atención es selectiva. La percepción tiene una función reguladora, en el sentido que filtra la información. (1995, p. 139)

Aspectos a los cuales Wittig agrega:

Los procesos sensoriales simplemente informan acerca de los estímulos ambientales; la percepción traduce estos mensajes sensoriales en forma comprensible. (1979, p. 72)

Desde un marco diferente, aunque complementario, la teoría de la atribución ha sido empleada para desplegar explicaciones acerca de las formas en las cuales se juzga de manera diferente a la gente. Dependiendo del significado que se atribuya al comportamiento de un individuo, se busca determinar si fue causado interna o externamente, condición que depende de: (1) si un individuo muestra diferentes comportamientos en distintas situaciones, (11) el grado de consenso acerca del tipo de respuesta común ante una situación similar, y (111) la consistencia referida de una respuesta en series de tiempo (Robbins, 1998).

Uno de los hallazgos más relevantes de la teoria de la atribución es que existen errores o prejuicios que distorsionan las atribuciones. La evidencia señala que cuando se hacen juicios acerca del comportamiento de otra gente, se tiende a subestimar la influencia de los factores externos y sobreestimar la de los factores internos o personales; lo que se denomina "error de atribución fundamental" (Robbins, 1998, p. 96). También hay una tendencia en los individuos a atribuir su propio éxito a los factores internos, como la habilidad o el esfuerzo, y el fracaso a factores externos denominado tendencia de "sesgo de auto-beneficio" (p. 96).

A partir de las teorias clásicas los investigadores buscan explicar el concepto de constancia perceptiva. Una vez que un objeto ha sido percibido como una entidad identificable, tiende a distinguirse como un objeto estable, de características permanentes, a pesar de las variaciones en su iluminación o distancia a la que aparece. Según la teoría

[...] la constancia en la percepción, al igual que la percepción de la profundidad y la mayoría de las percepciones, es resultado del individuo, de sintetizar las experiencias del pasado y las señales sensoriales presentes. (Hemann, 2003) 
Para la teoría de la percepción denominada Gestaltica (1920), "la percepción debe estudiarse no analizando unidades aisladas como las sensaciones simples, sino tomando en cuenta configuraciones globales" (Stanley et al., 1999, p. 305).

Existe una serie de factores que ayudan a dar forma o a distorsionar la percepción, los que pueden residir en el perceptor, cuando un individuo observa un blanco, tratará de interpretar lo que está viendo, pero esta interpretación estará condicionada por las características personales del perceptor, donde destacan la actitud, los motivos insatisfechos, los variados intereses personales, sus expectativas así como la experiencia, que también es un agente modificador de su apreciación. El objetivo o blanco afecta la percepción del observador, pues las características de los objetos o cosas observadas hacen que no siempre se vean de la misma forma, dentro de éstas las más importantes son el tamaño, la novedad, y el entorno. Por último, la situación, se refiere al contexto en el cual son observados los objetos, por lo que el entorno que rodea lo observado es sumamente importante. Dentro de estos factores se destacan el tiempo en que se desarrollan los hechos, el marco laboral y social de la situación.

En síntesis, estas teorías en su conjunto permitieron elaborar un instrumento de indagación para analizar la forma en que los docentes perciben el programa Escuelas Focalizadas Completas. Los diversos enfoques fueron útiles para la obtención de una visión adecuada de la percepción que estos tienen de las actividades como de los demás individuos que componen el programa. En particular, la teoría de la atribución sirvió para estudiar la percepción personal de los docentes acerca de los demás integrantes del programa, por otro lado, al cuantificar cómo perciben cada una de sus actividades y el programa en su conjunto se utilizó fundamentos de la teoría de la Gestalt así como los de las teorías clásicas.

En consecuencia, según la interpretación personal, basada en los distintos factores que influyen en la percepción y en sus modelos mentales, que de acuerdo a lo señalado por Peter Senge (1990) y otros como Goleman (1999), actúan como decodificadores de la realidad a la cual se tiene acceso, se puede decir entonces que los docentes, en el ámbito de la educación, seleccionan e interpretan la información disponible y perceptible de los eventos, características y resultados relacionados con la implementación del programa Escuelas Focalizadas Completas.

\section{Principales resultados}

\section{Considerandos metodológicos}

La relevancia de la investigación se fundamenta en que el docente es el actor más relevante del sistema desde el punto de vista de la intención de cambio, ello por cuanto la transformación de las prácticas pedagógicas es uno de los propósitos necesarios y funcionales para el éxito del Programa. En segunda instancia, los docentes son el principal recurso formativo, más aún en las escuelas focalizadas donde las carencias de capital social, económico, educacional del entorno aumentan la significación del docente para el logro adecuado de los objetivos curriculares. En tercer lugar, la percepción de este grupo respecto del Programa es un aspecto central para comprender lo realizado, así como obtener orientaciones para proponer adecuaciones en aquellos aspectos que requieran cambios.

El estudio se organizó en dos fases, una dirigida a explorar la temática en análisis, empleándose información secundaria y también primaria de tipo cualitativa de diversas fuentes; y otra, de tipo correlacional, usando información primaria mediante la aplicación de un instrumento tipo cuestionario-inventario. En esta última fase se definió la población objetivo del estudio, a saber: los docentes de las Escuelas Focalizadas Completas de la Provincia de Talca, cuya muestra provino de la variable "antigüedad del establecimiento en el P-900", 
obtenida mediante el indicador: "número de años de pertenencia al programa de una escuela focalizada, para lo cual se necesitaba registrar un valor igual o superior a uno".

El marco muestral correspondió a 831 docentes de este tipo de escuelas (datos provistos por la Secretaría Regional Ministerial de Educación), de los cuales 513 integran establecimientos que llevan más de un año en el programa. En la segunda fase del estudio se generaron dos grupos focales a los cuales fueron asignados aleatoriamente los participantes (sorteo simple sin reemplazo). Ambos grupos se conformaron con maestros de los mismos establecimientos, con la finalidad de mantener la homogeneidad de la muestra. Complementariamente se realizaron entrevistas a expertos: autoridades del sector de nivel provincial y supervisores técnicos de Escuelas Focalizadas.

Se aplicó un "muestreo probabilístico estratificado aproporcional”, para extrapolar los resultados a todos los estratos en cuestión. Se utilizó varianza máxima, un nivel de confianza de un 95\% y un margen de error del 6\%, la población objetivo alcanzó a 513 docentes con una muestra resultante de 176 profesores. Sin embargo, dado que en cinco comunas el número de docentes a entrevistar resultó inferior a 30 , se amplió la muestra para aproximar su relación a una población de distribución normal. Sin perjuicio de ello, el bajo número de casos del resto de las comunas no permitió aumentar la población significativamente, razón por la cual los resultados de esas comunas sólo serán incluidos en el análisis global de la población. De esta manera la muestra final quedó conformada por 249 docentes.

El instrumento utilizado fue un cuestionario elaborado a partir de información registrada en el estudio exploratorio, el que se organizó en cinco secciones. Las dos primeras corresponden a la indagación del conocimiento y metodología del programa; la tercera sección consulta los atributos y falencias para su implementación; la cuarta, recopila información sobre la eficacia del programa, y la quinta sección indaga sobre los factores que afectan la percepción de los docentes respecto del programa.

Se emplearon escalas likert y Nominal, por su facilidad de comprensión y además, porque permiten identificar categorías adecuadas para los eventos en estudio.

El análisis de datos emplea dos tipos de pasos: bivariado y análisis factorial, este último se utilizó para determinar el número y naturaleza de un grupo de constructos que subyacen en un conjunto de mediciones e identificar ideas fundamentales en la información.

\section{Antecedentes básicos del grupo}

La mayoría de los docentes entrevistados son mujeres (68\%), acordes con su proporción a nivel nacional. El rango modal de ejercicio profesional es de 20 a 29 años de docencia, representando un 42\%. Su porcentaje menor se ubica en el rango de menos de 10 años, con un 6\%, asimismo, la segunda frecuencia mayor en la demografía de la muestra está en el rango de 30 o más años, con un 34\%. Esto refleja que la cantidad de años de servicio de los docentes es alta ya que el 77\% está en un rango de 20 o más años de servicio, fenómeno común al país (MINEDUC, 2002) siendo ello crítico en algunas áreas de la docencia en enseñanza secundaria (Uribe, 2004)

En materia de edad, la mayor parte de los docentes están en el rango de los 50 o más años con un 46\%, mientras que la menor frecuencia es de los menores de 30 años con un 4\%. En consecuencia los docentes entre los 40 o más años representa un $86 \%$ de la muestra. Por lo tanto se puede afirmar que la edad promedio de los docentes es alta, cifra consistente con la de experiencia, materia que no es menor cuando hablamos de percepción.

\section{Conocimiento del programa}

La percepción del grado de conocimiento del programa se obtuvo mediante el análisis del conjunto de las variables para me- 
dir con mayor claridad sus dimensiones. Complementariamente se analizaron las respuestas según la comuna encuestada para comparar la realidad de las mismas.

El Cuadro $n^{\circ} 1$ muestra los resultados de las variables que conforman el grupo 1 (grado de conocimiento del programa). La mayor cantidad de respuestas corresponde a "de acuerdo" (37.9\%,) y la suma de esta alternativa con "muy de acuerdo" alcanza casi 65\%, lo que marca una tendencia mayoritaria. Las alternativas "en desacuerdo" y "muy en desacuerdo" suman un escaso 13.0\%.

Cuadro 1: Tiene gran conocimiento del programa de Escuelas Focalizadas

\begin{tabular}{|l|c|c|c|}
\hline \multicolumn{1}{|c|}{ Grupo 1 } & Frecuencia & \% Válido & $\%$ Acumulado \\
\hline Muy en desacuerdo & 106 & $6,08 \%$ & $6,08 \%$ \\
\hline En desacuerdo & 121 & $6,94 \%$ & $13,0 \%$ \\
\hline Ni acuerdo ni en desacuerdo & 389 & $22,32 \%$ & $35,3 \%$ \\
\hline De acuerdo & 661 & $37,92 \%$ & $73,3 \%$ \\
\hline Muy de acuerdo & 466 & $26,74 \%$ & $100,0 \%$ \\
\hline
\end{tabular}

Dada la fuerza de las alternativas "de acuerdo y muy de acuerdo", era razonable que en la mayoría de las comunas los resultados fuesen coincidentes. El hecho más llamativo corresponde a la comuna de Maule, donde sumadas las respuestas "muy de acuerdo" (61\%) y "de acuerdo" alcanzan un $88 \%$. Esta comuna es la que tiene el mayor grado de conocimiento del programa, seguida por las comunas de Talca (71\%) y de San Clemente (68\%). Destaca también la comuna de San Rafael, por el gran porcentaje de respuestas " $n i$ de acuerdo ni en desacuerdo" (49\%).

\section{Conocimiento de las metodologías}

Para determinar el grado de conocimiento de las metodologías se realizó una tabla de contingencia con los porcentajes de respuestas y la comuna encuestada, comparándose las realidades de las distintas comunas.

Los datos muestran que la alternativa "de acuerdo" es la preferida (37\%), valor que sumado a "muy de acuerdo" totalizan casi $60 \%$ de respuestas, lo que evidencia una tendencia dominante, permitiendo afirmar que un número relevante de docentes tiene un nivel significativo de conocimiento de las metodologías del programa. Las alternativas "muy en desacuerdo" y "en desacuerdo" suman 14.6\%, lo que confirma la afirmación anterior.

Cuadro 2: Tiene gran conocimiento de las metodologias empeladas en el prop
\begin{tabular}{|l|c|c|c|}
\hline \multicolumn{1}{|c|}{ Grupo 2 } & Frecuencia & $\%$ Válido & $\%$ Acumulado \\
\hline Muy en desacuerdo & 165 & $4,4 \%$ & $4,4 \%$ \\
\hline En desacuerdo & 379 & $10,2 \%$ & $14,6 \%$ \\
\hline Ni de acuerdo ni en desacuerdo & 966 & $25,9 \%$ & $40,5 \%$ \\
\hline De acuerdo & 1377 & $36,9 \%$ & $77,4 \%$ \\
\hline Muy de acuerdo & 848 & $22,70 \%$ & $100,0 \%$ \\
\hline
\end{tabular}

El análisis a nivel de las comunas ratifica la tendencia positiva, ya que las respuestas "de acuerdo" y "muy de acuerdo" completan más del $50 \%$ de las selecciones en cinco comunas. Las restantes comunas de la provincia (cinco) tienen niveles similares de conocimiento y no muestran grandes diferencias entre las alternativas, aunque en la de San Rafael la alternativa "ni de acuerdo ni en desacuerdo" sobrepasa el 50\% de las preferencias, lo que indica consistencia en la medición y expone un menor conocimiento de las metodologías del programa.

\section{Atributos y falencias del Programa}

Para identificarlas se realizó un análisis por cada variable en forma independiente, con la finalidad de alcanzar mayor profundidad y especificación.

\section{Atributos}

En esta condición se clasificaron las variables que muestran una tendencia dominante como atributo positivo, a saber:

- "Interés en las actividades por parte de los alumnos”, el 56\% de los docentes señala que son interesantes, un $4 \%$ piensa lo contrario y un $39 \%$ es indiferente. 
- “Autonomía de trabajo", el 50\% de los maestros está de acuerdo en que el programa lo permite, un $12 \%$ no está de acuerdo y al $38 \%$ le es indiferente.

- "Existencia de vías alternativas para enseñar", el 55\% de los docentes está de acuerdo en que el P-900 cumple esta condición, en tanto que al $37 \%$ le es indiferente. La respuesta con la mayor frecuencia es la alternativa "de acuerdo" (41\%), lo que muestra una clara tendencia para clasificarla como atributo.

- "Existencia de buena comunicación escuelafamilia-alumno", las opciones "de acuerdo" y "muy de acuerdo" suman 58\% de las preferencias, siendo la más elegida la alternativa "de acuerdo" con un $41 \%$.

- "Ambiente entre profesores y alumnos", calificado por el 53\% como afirmativo, en tanto un 7\% piensa lo contrario.

- "Pasantías a los profesores", las alternativas "de acuerdo" y "muy de acuerdo" representan el 49\%, alcanzando la primera preferencia, sin embargo, la alternativa "ni de acuerdo ni en desacuerdo" con un 31\% de preferencias, inciden en una interpretación compleja de esta variable, que si bien no evidencia una tendencia clara, muestra que las respuestas tienden hacia el lado positivo, por lo que esta variable puede ser más un atributo que una falencia, sin tendencia claramente definida.

- Finalmente, "apoyo del supervisor", un 45\% señala afirmativamente esta condición, el 20\% indica que no y al 35\% le es indiferente.

\section{Falencias}

En general resultó relevante la respuesta al ítem "el programa aumenta la carga de trabajo" con un 52\% de frecuencia, aunque al 35\% le fue indiferente, y un 14\% no está de acuerdo con ella. En consecuencia es clasificada como falencia.

\section{Resultados sin tendencia definida}

Corresponden a esta categoría aquellas respuestas que no tuvieron una inclinación manifiesta como atributo o falencia, es decir su tendencia es la no tendencia polar.

- Un 40\% de los docentes entrevistados considera que las pautas de trabajo son claras, aunque el $42 \%$ se muestra indiferente y el 18\% opina negativamente: no son claras.

- Un 42\% de los maestros indica que existen las instancias adecuadas para resolver dudas, mientras que al 37\% tiene indiferencia y el $21 \%$ está en desacuerdo con esta afirmación. - El 21\% afirma que el programa es de fácil comprensión, lo que es negado por el 35\% de los docentes. A un $44 \%$ le es indiferente, representando la alternativa de mayor preferencia. - Del mismo modo, un 45\% cree que el programa quita tiempo, el 37\% es indiferente en este ítem y el 18\% está en desacuerdo con lo señalado.

- Respecto de si cuenta con material adecuado, un $41 \%$ lo confirma, lo niega un 18\%, y al $42 \%$ le es indiferente. A pesar de que existe una tendencia hacia las respuestas positivas, no logra un porcentaje mayoritario de las respuestas. - Complementariamente, las respuestas acerca de si existe material inutilizado, el 36\% señaló estar de acuerdo o muy de acuerdo, en tanto un 39\% indicó "ni de acuerdo ni en desacuerdo" siendo la más seleccionada.

- Las opiniones respecto de la utilidad de las reuniones informativas, presenta una alta frecuencia para "ni de acuerdo ni en desacuerdo" (41\%), las alternativas "de acuerdo" y "muy de acuerdo" suman 27\%, y "en desacuerdo" y "muy en desacuerdo" el 33\% de las preferencias, sin representar tampoco una mayoría significativa.

- La existencia de retroalimentación entre docentes y el ministerio, fue contestada afirmativamente por un $27 \%$, mientras que "en desacuerdo" y "muy en desacuerdo" lo hace un $42 \%$, no mostrando dominio absoluto de las respuestas.

- La existencia de atención individualizada para casos especiales, un tercio de los encuestados "no está de acuerdo ni en desacuerdo". Las otras alternativas representan proporciones similares y coinciden con lo señalado. En consecuencia, 
no es posible hacer una definición clara y definitiva al respecto.

- La existencia de una cuenta pública de los resultados del programa, implica para el 32\% "ni de acuerdo ni en desacuerdo". Las respuestas "de acuerdo" o "muy de acuerdo" suman 30\% y "en desacuerdo" o "muy en desacuerdo" 38\%. Por lo tanto no se puede definir de manera fehaciente si esta variable es un atributo o una falencia.

- En el otorgamiento de medios a los padres para facilitar el aprendizaje en el hogar, las opciones "de acuerdo" y "muy de acuerdo" suman 37\% y la alternativa "ni de acuerdo ni en desacuerdo" 32\%.

\section{Síntesis y discusión}

Entre los atributos percibidos como positivos destacan dos aspectos centrales vinculados con la estrategia impulsada por el P-900. El primero corresponde al interés de los alumnos por las actividades del Programa, siendo un factor de atracción motivacional relevante, clave para instalar procesos de cambio, en consecuencia se trata de un factor clave e importante, positivamente percibido. El segundo se refiere al desarrollo de la autonomía en el trabajo, elemento que tiene un doble papel; por un lado es una competencia clave para el éxito de las personas en su desempeño presente y futuro en una sociedad cada vez más indeterminada y demandante de autonomía de las personas, segundo, para el caso de los docentes, implica un factor de reconversión laboral de significación, aún no percibido del todo por ellos como significativo, dadas las respuestas que se comentan más adelante.

Las restantes variables que son percibidas como positivas tienen un rol sinérgico con los objetivos del P-900, y se orientan al mejoramiento del apoyo en el hogar y reforzamiento de la capacidad de los docentes. Complementariamente, la percepción de un factor negativo como "más trabajo de parte de los docentes" puede ser revertida con un adecuado reconocimiento de esta dedicación sin que implique, aunque no debiese excluir, tampoco, lo pecuniario.

Los resultados muestran un desafío importante, que es conducir la valorización positiva de aquellas percepciones "de indiferencia" de algunas dimensiones. Esta se dirige - en lo fundamental - al área de trabajo docente del P-900. Primero buscando que existan pautas y criterios más claros de trabajo, es decir un camino más definido que oriente a los docentes en algunos ámbitos en los que hay incertidumbre, lo que es muy significativo como petición, de manera que la respuesta que se entregue debe permitir reforzar su profesionalismo y - paralelamente responder a esta situación; y el segundo ámbito tiene que ver con las relaciones de los docentes con el Ministerio, los supervisores y la retroalimentación de su trabajo, materia crucial que puede ser mejorada con claros beneficios de eficiencia de los resultados.

\section{Eficiencia del Programa}

Se realizó un análisis por items que permitió definir los aspectos mejor evaluados y que, según la percepción de los docentes, fueron logrados satisfactoriamente. Las variables empleadas son las definidas en el proyecto original para la evaluación de su eficacia.

\section{Aspectos logrados}

Los principales aspectos que se han clasificado como logrados son:

- La "facilitación del aprendizaje" resultó la alternativa mejor evaluada, obteniendo la opción "de acuerdo" un 47\% de las preferencias, a que se suman los "muy de acuerdo" con un 64\% de apoyo.

- El "mejoramiento de la convivencia escolar", con 53\% "de acuerdo" o "muy de acuerdo". La segunda elección es "ni de acuerdo ni en desacuerdo" (38\%).

- En referencia a la "ayuda de alumnos rezagados”, el 56\% está “de acuerdo” o "muy 
de acuerdo", lo que muestra una tendencia mayoritaria hacia la respuesta positiva en esta variable. El 33\% respondió "ni de acuerdo ni en desacuerdo".

- Por otra parte, la "mejora de las calificaciones de lenguaje", da cuenta que la alternativa "ni de acuerdo ni en desacuerdo" obtuvo la mayor frecuencia (41\%), sin embargo, "de acuerdo" y "muy de acuerdo" representan en total 56\% de las preferencias, por lo que la tendencia al respecto es mayoritaria y positiva. Esto permite interpretar que la variable "mejora de las calificaciones de lenguaje" fue satisfactoriamente cumplida por el proyecto.

- Finalmente, si “el programa hace de los profesores agentes participativos de los nuevos métodos de educación", el 60\% respondió "de acuerdo" o "muy de acuerdo".

\section{Ítems sin tendencia dominante}

- En relación al "mejoramiento del ambiente familiar de los alumnos", aunque el 49\% está "de acuerdo" o "muy de acuerdo", comparte las preferencias con "ni de acuerdo ni en desacuerdo" (37\%), lo que tampoco representa una tendencia mayoritaria, eso sí positiva, los indecisos son muchos.

- La "mejora de las calificaciones de matemáticas", tuvo resultados diferentes a su análoga de lenguaje. La alternativa con mayor preferencia fue "ni de acuerdo ni en desacuerdo" (46\%), mientras que sumadas las alternativas "de acuerdo" o "muy de acuerdo" alcanzan 48\%, respuestas que se asemejan mucho.

- Respecto de la "motivación de los alumnos para estudiar", el 43\% está "de acuerdo" o "muy de acuerdo" y 44\% "ni de acuerdo ni en desacuerdo", por lo que no es posible hacer una inferencia definitiva acerca de esta variable. - En cuanto al "mejoramiento de las capacidades analíticas de los alumnos", el mayor número de repuestas (43\%) lo registró "ni de acuerdo ni en desacuerdo", mientras que las alternativas "de acuerdo" o "muy de acuerdo" suman un $43 \%$, lo que no permite hacer una afirmación definitiva del grado de logro del mismo.

- En relación al "mejoramiento de las capacidades de comprensión de los alumnos", la alternativa más elegida fue "ni de acuerdo ni en desacuerdo" con un $45 \%$, mientras que "de acuerdo" o "muy de acuerdo" suman un 44\%, dando cuenta de una tendencia compartida.

\section{Síntesis y discusión}

Los resultados sobre la percepción de la dimensión eficiencia son consistentes con la percepción de los atributos del Programa, en razón de ello existe un factor estructural en estos aspectos que constituye un capital aprovechable de manera más intensiva, con políticas ad hoc. Esta coherencia se expresa tanto en el plano de los objetivos o fines, como en las metodologías de trabajo del P-900. Adicionalmente, confirman lo señalado los resultados de las dimensiones de motivación, de formas de colaboración con estudiantes rezagados, como también la incorporación de nuevas metodologías docentes.

$\mathrm{Al}$ igual que en la sección anterior, hay un campo de desafíos muy "positivos de alcanzar" que corresponde a objetivos pedagógicos trascendentes, cuyo dominio implicaría un logro de proporciones del Programa: el hecho de mejorar la motivación de los estudiantes por el aprendizaje y de incrementar sus capacidades analíticas, son dos competencias ejes en el desarrollo del pensamiento.

\section{Análisis jerarquizado de las respuestas}

Resumiendo el examen acerca de cómo influyen diversas variables en la percepción de los docentes, se realizó un análisis por ítem/variable, que implicó su jerarquización mediante el método de Thurstone ${ }^{5}$, verificándose cuáles fueron las

5. Procedimiento que examina si los valores escalares de los estímulos responden a una escala de intervalos asimilable a una distribución normal. Esta distribución, que no es directamente observable, recibe el nombre de "distribución discriminativa" (Barbero, 1993; Meliá, 1990). 
más importantes respecto de la percepción del programa por parte de los docentes.

Los docentes perciben la variable "confianza en los programas de educación" como la más importante, seguida de "satisfacción con las condiciones de trabajo" y "logro de buenos resultados".

Por su parte, las variables menos importantes, según orden de menos a más son "búsqueda del desarrollo profesional", seguida por "logro de un mejor ambiente de trabajo" y "necesidad de reducir el número de alumnos por sala".

Los resultados se inscriben en lo comentado. La percepción de Confianza en el Programa se transforma en un capital relevante para cualquier empresa que apunte a objetivos trascendentes. Llama la atención que junto a esta condición y otras que son consistentes con el desarrollo profesional docente, jerarquicen como aspecto menos logrado el desarrollo profesional. El P-900 ha desarrollado en los docentes nuevas pautas de desempeño profesional, distintas de las que tradicionalmente son percibidas como "profesionales" y no ha validado adecuadamente los criterios de desempeño de la nueva propuesta de hacer metodológico, de forma que los docentes no perciben como estándar de desempeño adecuado el cambio profesional experimentado.

\section{Análisis de factores de componentes principales}

El análisis de componentes principales definió 15 factores mediante el método Varimax de rotación de datos. La confiabilidad de cada factor dio un resultado de 0.95 con todas las variables. En consecuencia, el total de variables y factores reúne información consistente $y$, además, el modelo resultante permite explicar un $72 \%$ de la varianza total.

Los factores que no presentan un valor mínimo aceptable para el análisis (equivalente a 0.7) que sustente obtener información consistente fueron los numerales: 10 a 12, 14 y 15 , a lo cual se agrega el factor 14 que cuenta con una sola variable, lo que hace imposible calcu- lar su confiabilidad, por lo que este factor fue considerado como poco confiable. En razón de ello, los factores efectivamente objeto de análisis cuentan con una confiabilidad mayor a un $70 \%$. Después de reducir los factores poco confiables y ratificando los 10 más consistentes, se alcanzó un nivel de confiabilidad total del modelo de 0.9497, que es considerado adecuado.

La revisión de los factores da cuenta de su amplia estructura, que considera aspectos operacionales y propios de la gestión educativa hasta temas de conocimiento y apropiación de las metodologías. Destaca en primer lugar el factor Eficacia, que se refiere a los propósitos y fines del P-900, traduciéndose en las operaciones y su orientación a resultados, lo que se evidenciaría en las evaluaciones realizadas al Programa por las pruebas SIMCE. La varianza explicada por este factor $(28 \%)$ es importante y representa algo menos de la mitad de la varianza total explicada del conjunto de factores, lo que expresa con mayor fuerza su peso.

En segundo lugar ingresa el factor Conocimiento del programa, que corresponde a la difusión y operaciones del mismo, tiene la posibilidad de ser manipulado positivamente por la autoridad de manera que sea más eficiente y produzca mayores efectos positivos. Es decir, es instrumentalmente más asequible que el anterior.

Un tercer nivel de interpretación implica revisar los factores atributos y falencias relacionados con el desarrollo profesional, variable que incide en la percepción. Esta área, que ha sido percibida en su parcialidad como positiva, lograda y eficiente, en la suma total no alcanza a estructurar un sistema perceptivo que refuerce el cambio, tarea que está pendiente y que potenciaría los resultados alcanzados.

Finalmente, es interesante constatar que con la gestión de los cinco primeros factores, a saber: Eficacia; Conocimiento del programa; Administración del programa; Conocimiento de la responsabilidad del programa, y Atributos y falencias relacionados con el desarrollo profesional, se puede explicar casi el 50\% de la varianza total y prácticamente el $80 \%$ de la 
Cuadro 3: Análisis de factores de componentes principales (según aportes)

\begin{tabular}{|c|c|c|c|c|}
\hline FACTOR & VARIABLES & Confiabilidad & $\begin{array}{l}\text { Varianza } \\
\text { Explicada }\end{array}$ & $\begin{array}{c}\text { Varianza } \\
\text { acumulada }\end{array}$ \\
\hline Factor 1: Eficacia & $\begin{array}{l}\text { 4.1 Facilitación del aprendizaje. } \\
\text { 4.2 Mejora de las calificaciones de lenguaje. } \\
\text { 4.3 Mejora de las calificaciones de matemáticas. } \\
\text { 4.4 Motivación personal para estudiar de los alumnos. } \\
\text { 4.5 Mejoramiento de las capacidades analíticas de los alumnos. } \\
\text { 4.6 Mejoramiento de las capacidades de comprensión de los alumnos. } \\
\text { 4.7 Mejoramiento de la convivencia escolar. } \\
\text { 4.8 Ayuda de alumnos rezagados. } \\
\text { 4.9 Mejoramiento del ambiente familiar de los alumnos. } \\
\text { 4.10 El programa hace de los profesores agentes participativos } \\
\text { de los nuevos métodos de educación. }\end{array}$ & 0.9231 & 27.5839 & 27,5839 \\
\hline $\begin{array}{l}\text { Factor 2: Conocimiento } \\
\text { del programa }\end{array}$ & $\begin{array}{l}\text { 1.1 conoce claramente los objetivos del programa. } \\
1.2 \text { está informado de cómo la escuela ingresa al programa } \\
1.3 \text { está informado de cómo la escuela sale del programa } \\
1.4 \text { conoce los talleres de profesores } \\
\text { 1.6 está informado que el ministerio aporta una mayor cantidad } \\
\text { de material de trabajo } \\
1.7 \text { está informado del desempeño que se espera de mí } \\
2.1 \text { se como realizar las distintas actividades que posee el programa } \\
2.2 \text { se como utilizar los materiales aportados por el programa. }\end{array}$ & 0.9080 & 7.6840 & 35,2678 \\
\hline $\begin{array}{l}\text { Factor 3: Administración } \\
\text { del programa }\end{array}$ & $\begin{array}{l}2.6 \text { conozco como se desarrollan las jornadas de gestión } \\
2.7 \text { se como se coordina el programa } \\
2.8 \text { se como se desarrolla el programa } \\
2.9 \text { conozco como se evalúa el programa } \\
3.1 \text { están claras las pautas de trabajo } \\
3.2 \text { existen instancias adecuadas para resolver dudas } \\
3.13 \text { son suficientes las reuniones informativas para discutir los } \\
\text { alcances del proyecto }\end{array}$ & 0.9103 & 5.7203 & 40,9882 \\
\hline $\begin{array}{l}\text { Factor 4: Conocimiento } \\
\text { de la responsabilidad del } \\
\text { programa }\end{array}$ & $\begin{array}{l}\text { 2.10 debe otorgar medios a los padres para facilitar el } \\
\text { aprendizaje en el hogar } \\
2.11 \text { debe tener contacto con los padres por medio de citaciones } \\
2.12 \text { debe realizar actividades formales con alumnos y apoderados } \\
2.13 \text { debo mantener informadas a las familias con los progresos } \\
\text { de sus hijos } \\
2.14 \text { se cono se realizan las ayudantías entre el monitor y los alumnos } \\
2.15 \text { debo mantenerme informado de los avances que logra el } \\
\text { monitor con los alumnos } \\
\text { 3.19 existe una buena comunicación escuela-familia-alumno }\end{array}$ & 0.8147 & 4.5468 & 45,5350 \\
\hline $\begin{array}{l}\text { Factor } 5 \text { : Atributos y } \\
\text { falencias relacionadas } \\
\text { con el desarrollo } \\
\text { profesional }\end{array}$ & $\begin{array}{l}\text { 3.14 existe retroalimentación entre las observaciones de los } \\
\text { docentes y el ministerio } \\
3.15 \text { existe atención individualizada para casos especiales } \\
3.16 \text { se realizan pasantías de profesores } \\
3.17 \text { se da cuenta publica de los resultados del programa } \\
3.18 \text { se otorgan medios a los padres para facilitar el aprendizaje } \\
\text { en el hogar }\end{array}$ & 0.8295 & 3.8447 & 49,3797 \\
\hline $\begin{array}{l}\text { Factor 6: Conocimiento } \\
\text { apoyo externo } \\
\text { metodología }\end{array}$ & $\begin{array}{l}2.3 \text { se como se desarrollan las reuniones del taller de profesores } \\
2.4 \text { se utilizar apropiadamente los recursos de la biblioteca del profesor } \\
2.5 \text { se como funcionan las asesorías del supervisor } \\
3.6 \text { conozco como se desarrollan las jornadas de gestión }\end{array}$ & 0.7962 & 3.3690 & 52,7486 \\
\hline $\begin{array}{l}\text { Factor } 7 \text { : Variables que } \\
\text { afectan la percepción }\end{array}$ & $\begin{array}{l}5.2 \text { usted es un agente participativo en su trabajo } \\
5.3 \text { se siente comprometido con los resultados de la escuela } \\
5.9 \text { constantemente participa de cursos de perfeccionamiento } \\
5.10 \text { busca una estabilidad laboral } \\
5.11 \text { desea desarrollarse como profesional } \\
5.12 \text { busco una estabilidad económica en el trabajo }\end{array}$ & 0.7966 & 3.1799 & 55,9285 \\
\hline $\begin{array}{l}\text { Factor 8: Atributos y } \\
\text { falencias relacionadas } \\
\text { con el trabajo }\end{array}$ & $\begin{array}{l}\text { 3.3 el programa es de fácil comprensión; } \\
\text { 3.4 el programa aumenta la carga de trabajo } \\
\text { 3.5 el programa quita tiempo a las demás actividades }\end{array}$ & 0.7045 & 2.7483 & 58,6768 \\
\hline $\begin{array}{l}\text { Factor 9: Atributos y } \\
\text { falencias relacionadas } \\
\text { con la docencia }\end{array}$ & $\begin{array}{l}\text { 3.11 existen vias alternativas para enseñar los contenidos del programa } \\
\text { 3.12 el programa crea un mejor ambiente entre profesores y alumnos }\end{array}$ & 0.7396 & 2.1483 & 60,8251 \\
\hline $\begin{array}{l}\text { Factor 13: Atributos y } \\
\text { falencias relacionadas con } \\
\text { el apoyo de recursos fisicos }\end{array}$ & $\begin{array}{l}3.7 \text { se cuenta con el material adecuado } \\
\text { 3.9 cuenta con el apoyo necesario del supervisor } \\
\text { 3.10 el programa da autonomía para trabajar }\end{array}$ & 0.7296 & 1.8163 & 62,6415 \\
\hline
\end{tabular}


explicada por el modelo, de forma que aplicando el principio de parsimonia, son de significación neta para un estudio en este ámbito.

Esta evidencia permite destacar además que, de acuerdo con las percepciones de los docentes considerados en el estudio, el impacto en los ámbitos social y educacional del P-900 se asocia en medida importante con la percepción de su eficacia y de un adecuado nivel del conocimiento del Programa, y también de sus metodologías, criterios y procedimiento de operación.

\section{Consideraciones finales}

\section{Resultados}

El análisis de la percepción de los docentes participantes en el Programa P-900 corresponde a un grupo dominante de género femenino $(67 \%)$ y con edades que sobrepasan los 50 años y superan los 30 de servicio docente. Es decir, corresponde a un grupo que posee vasta experiencia sobre la materia, lo que implica que su percepción será la resultante de un contraste con interesantes prácticas. De igual forma se señaló que el programa está siendo implementado en todas las comunas de la provincia de Talca con elevados índices de información en las comunas.

La autocalificación de un alto grado de conocimiento del Programa de parte de los docentes es una respuesta que concentró la mayoría de las opiniones de los participantes; punto que se extiende en términos similares al conocimiento de los aspectos metodológicos. Es decir, los docentes asumen que estos aspectos "externos" están incorporados, en grado importante a su actuar: conocen el programa y su metodología.

En razón de eso, la percepción de los atributos positivos mencionados por los docentes es consistente con ello. Primero porque evidencian el gran interés de los alumnos por las actividades del Programa, y segundo porque indican que ha incidido en el desarrollo de la autonomía en el trabajo, lo que conforma una competencia clave de éxito para su desempeño futuro. A su vez, para el docente implica un factor de desarrollo laboral de significación, que aún no es debidamente percibido por sí mismo en la importancia que reviste. Las restantes variables valuadas como positivas tienen un rol sinérgico con los objetivos del P-900 dando cuenta del hecho que los docentes poseen una percepción, en general muy positiva del Programa y sus efectos en los estudiantes y algo menos en ellos mismos.

Como puede comprenderse la percepción del factor "más trabajo de parte de los docentes" como negativa, es bastante razonable, toda vez que un proceso de esta naturaleza implica más esfuerzo, en lo fundamental frente al formato tradicional, algo que los docentes quieren destacar pues ha sido un gremio tradicionalmente postergado, poco reconocido socialmente (Núñez, 2003).

La percepción de la dimensión eficiencia vuelve a ser plenamente convergente con la referida a los atributos del Programa, en razón de ello hay clara sinergia en estos aspectos, la que se expresa en el plano de los objetivos o fines, como en las metodologías de trabajo del Programa, confirmándose lo señalado con las variables referidas a motivación, trabajo con estudiantes rezagados e incorporación de nuevas metodologías docentes.

Todo lo señalado se sintetiza en una percepción de gran confianza en el Programa dando cuenta además que existe una percepción positiva sobre el hacer del programa y su eficiencia en los puntos estratégicos más importantes del mismo.

\section{Desafíos}

El principal desafío del Programa es analizar la posibilidad de su rediseño, teniendo en cuenta dos factores cruciales, el primero ya plenamente identificado y tratado, pero aún sin plena solución, es el problema del "lento egreso de muchos establecimientos escolares", el segundo está asociado a los resultados de este estudio: la necesidad de 'convertir' las percepciones que 
están en el intervalo de "ni uno ni otro aspecto" y llevarlas al ámbito de lo positivo.

Ello es plenamente posible pues existe una muy buena percepción en los docentes de los aspectos centrales que conforman el núcleo esencial del P-900. En consecuencia, se dispone una base sólida y pertinente a partir de la cual se pueden plantear propuestas de intervención adecuadas.

En segundo lugar, los docentes tienen una percepción positiva de su rol, aunque éstos destacan que han debido pagar algunos costos que no son de su responsabilidad, entre los que se mencionan el incremento del trabajo. Lo cierto es que este factor puede ser redireccionado hacia el ámbito positivo con una política más clara de reconocimiento e incentivo a los maestros que están en estas escuelas. Ello es un elemento indiscutible de una política que se basa en la acción positiva, es que emplee la 'acción positiva' con los docentes que trabajan en el mismo Programa. La adecuada resolución de esta traba - una de las de mayor complejidad de la práctica docente $^{6}$ - permitirá una capitalización en el ámbito de la profesionalización docente, aspecto significativo en los cuales los maestros han dado muestras de avances relevantes aunque sienten más la "pérdida" que las "ganancias", en lo fundamental porque los criterios de medición al respecto - o los parámetros bajo los cuales se les evalúa - resultan obsoletos al tenor de las exigencias presentes y futuras.

Teniendo claro que la estrategia de éxito del P-900 está estrechamente asociada con las prácticas docentes, los resultados muestran que los maestros tienen buena disposición al desarrollo de prácticas más significativas: autonomía de los aprendizajes, estimulo y motivación a los estudiantes, fomento de la diversidad y apoyo a los más desvalidos y uso de metodologías innovadoras.

Complementariamente, la percepción de los docentes no hace del todo justicia al grado y profundidad de las aportaciones de los estudiantes que participan del programa y del compromiso que supone el trabajo de sí mismos como agentes activos de su propio proceso de desarrollo educacional, ello porque los maestros tienden a valorar más su esfuerzo que el de los demás, lo que es algo propio de la Teoría Atribucional (como se señaló en la cuarta sección de este artículo).

En la línea del mejoramiento y fundamentalmente del rediseño del Programa, todo el ámbito con la familia puede ser potenciado en mejor forma. Sin embargo, aquello referido a la labor del Ministerio de Educación, a las normativas propias del Programa y al apoyo de los supervisores es un área que requiere una reingeniería pues su mejor percepción potenciaría los resultados del Programa en todos sus ámbitos.

Este aspecto es un tema crucial para las estrategias de focalización mediante acción positiva. El Ministerio y sus agentes deben tener un sistema de información muy ágil en estos ámbitos que permita apoyar las iniciativas docentes y que estimule su desarrollo, proceso que debe ser casi simultáneo con los resultados que se consiguen, lo que es difícil de alcanzar con estructuras ministeriales pesadas.

En síntesis, comprendiendo que la estrategia de acciones positivas y políticas focalizadas son herramientas limitadas a situaciones específicas, y por ende no deben emplearse como instrumentos permanentes y de amplio alcance, los resultados derivados de este estudio dan orientaciones en materia de la percepción de los docentes, confirman la adecuada predisposición para impulsar políticas que sean más eficientes y muestran que queda espacio en estos momentos para que las autoridades del sector, mediante medidas simples, apunten a reconocer en mejor forma el trabajo de los docentes y, a su vez mejoren los mecanismos de comunicación entre docentes, autoridades y supervisores, como asimismo refuercen el trabajo con materiales más adecuados que permitan potenciar debidamente el Programa y aumentar significativamente sus resultados.

6. Existe la constante creencia en los maestros que su labor está descompensada de sus retribuciones económicas. En consecuencia la sociedad tiene una 'deuda' casi de carácter histórico que no se salda fácilmente. 
Las políticas educativas focalizadas, centradas en acciones positivas están llamadas a cumplir un rol determinante, en períodos reducidos de tiempo, si son debidamente apoyadas y si cuentan con mecanismos eficien- tes de adecuación, en caso contrario su vida útil se reduce. El P-900 es un Programa que ha cumplido exitosamente un largo ciclo pero requiere un rediseño profundo, algunos de estos aspectos se derivan de la percepción de los docentes.

\section{Referencias bibliográficas}

AAKER, D.; DAY, G. Investigación de mercados. Tercera Edición. Mc Graw-Hill. 1989.

ARNO, W. Introducción a la psicología, Mc Graw-Hill latinoamericana.

AYLWIN, M. Los programas focalizados. En: HEVIA, R. (Ed.) La educación en Chile hoy. Santiago: Ediciones Universidad Diego Portales. 2003. p. 151-164.

BARBERO, M. I. Psicometría II: métodos de elaboración de escalas. Madrid: UNED. 1993.

BURKE, W. W. Desarrollo organizacional: punto de vista normativo. Argentina: Addison-Wesley Iberoamericana. 1988.

COX, C. Las políticas educacionales de Chile en las últimas dos décadas del siglo XX. En: COX, C. (Ed.) Políticas educacionales en el cambio de siglo: la reforma del sistema escolar en Chile. Santiago: Editorial Universitaria. 2003. p. 19-114.

DIPRES Estudio Programa de escuelas de Sectores Pobres (P-900). Ministerio de Hacienda, Dirección de Presupuesto. Consultado el 01/12/04. 2002.

GARCÍA-HUIDOBRO, J. E.; SOTOMAYOR, C. La centralidad de la escuela en la política educativa de los noventa. En: COX, C. (Ed.). Políticas educacionales en el cambio de siglo: la reforma del sistema escolar en Chile. Santiago: Editorial Universitaria, 2003. p. 253-315.

GONZÁLEZ, P. Estructura Institucional, recursos y gestión en el sistema escolar chileno. En: COX, C. (Ed.). Políticas educacionales en el cambio de siglo: la reforma del sistema escolar en Chile. Santiago: Editorial Universitaria, 2003. p. 597-660.

HERNÁNDEZ, R.; FERNÁNDEZ, C.; BAPTISTA, P. Metodología de la investigación. Segunda Edición. Mc Graw-Hill. 1991.

KINNEAR, T.; TAYLOR, J. Investigación de mercados. Quinta Edición. Mc GRAW-Hill. 1998.

LAMBIN, J. Marketing estratégico. Segunda Edición. Mc Graw-Hill. 1995.

MELIÁ, J. L. Métodos de escalamiento unidimensional. Valencia: Cristóbal, Serrano. 1990.

MINEDUC. En la ruta del autoperfeccionamiento. Revista de Educ. Edición, n. 289, 2001. p. 4-5.

MINEDUC. Boletín Informativo P-900. Gobierno de Chile. Santiago de Chile. 2002. p. 1-6

MINISTERIO de Educación. Octubre 2003. Disponible en: http://www.simce.cl. Acceso en: marzo 2004.

NÚÑEZ, I. El profesorado, su gremio y la reforma de los años noventa: presiones de cambio y evolución en la cultura docente. En: COX, C. (Editor). Políticas educacionales en el cambio de siglo: la reforma del sistema escolar en Chile. Santiago: Editorial Universitaria, 2003, p. 455-518.

OCDE (Organización para la Cooperación y el Desarrollo) Chile: revisión de las políticas nacionales de educación. Paris. 2004.

ROBBINS, S. Comportamiento organizacional. Octava Edición. Mc Graw-Hill. 1998. 
RUBIO GONZÁLEZ, M. A.; ROJAS, P. H. A. Percepción de los docentes participantes del Programa Escuelas Focalizadas Completas de la provincia de Talca. Facultad de Ciencias Empresariales, Universidad de Talca. 2004.

SENGE, P. La quinta disciplina. Barcelona: Vergara Granica. 1990.

SOTOMAYOR, C. Programa de mejoramiento de la calidad de escuelas básicas de sectores pobres (P-900). En: GARCíA HUIDOBRO, J. E. (Ed.). La reforma educacional chilena. Madrid: Popular, 1999. p. 69-90.

STANLEY, C.; LAWRENCE, W.; JAMES, E. Sensación y percepción. Tercera Edición. Mc Graw-Hill. 1999.

URIBE, D. Oferta educativa y oferta de graduados de educación superior. En: BRUNNER, J. J.; MELLER, P. Oferta y demanda de profesionales y técnicos en Chile. Santiago: RIL Editores. 2004, p. 131-170.

WITTIG, A. Introducción a la psicología. Primera Edición. Mc Graw-Hill. 1979.

Recebido em 20.07.05

Modificado em 03.01 .06

Aprovado em 15.03.06

Miguel Bustamante es Economista y Dr. en Ciencias Económicas y Empresariales, Académico de la Facultad de Ciencias Empresariales de la Universidad de Talca. mabu@utalca.cl

Sebastián Donoso es Educador y Dr. en Educación, Académico del Instituto de Investigación y Desarrollo Educacional de la misma universidad. 